\title{
Corrigendum: Selenomethionine
} Improves Mitochondrial Function by Upregulating Mitochondrial Selenoprotein in a Model of Alzheimer's Disease

\author{
Chen Chen ${ }^{1,2}$, Yao Chen ${ }^{1}$, Zhong-Hao Zhang ${ }^{1}$, Shi-Zheng Jia ${ }^{1}$, Yu-Bin Chen ${ }^{1}$, \\ Shao-Ling Huang ${ }^{1}$, Xin-Wen $\mathrm{Xu}^{1}$ and Guo-Li Song ${ }^{1,3,4 *}$
}

${ }^{1}$ Shenzhen Key Laboratory of Marine Bioresources and Ecology, College of Life Sciences and Oceanography, Shenzhen University, Shenzhen, China, ${ }^{2}$ College of Physics and Optoelectronic Engineering, Shenzhen University, Shenzhen, China, ${ }^{3}$ Shenzhen Bay Laboratory, Shenzhen, China, ${ }^{4}$ Shenzhen-Hong Kong Institute of Brain Science-Shenzhen Fundamental Research Institutions, Shenzhen, China

Keywords: selenomethionine, mitochondrial selenoprotein, Alzheimer's disease, mitochondria dysfunction and dementia, therapeutic effect and mechanism

\section{A Corrigendum on}

OPEN ACCESS

Approved by:

Frontiers Editorial Office,

Frontiers Media SA, Switzerland

*Correspondence:

Guo-Li Song

lilys@szu.edu.cn

Specialty section:

This article was submitted to

Cellular and Molecular Mechanisms of

Brain-aging,

a section of the journal

Frontiers in Aging Neuroscience

Received: 15 November 2021

Accepted: 16 November 2021

Published: 20 December 2021

Citation:

Chen $C$, Chen Y, Zhang Z-H, Jia S-Z,

Chen $Y$-B, Huang $S-L, X U X-W$ and

Song G-L (2021) Corrigendum:

Selenomethionine Improves

Mitochondrial Function by

Upregulating Mitochondrial

Selenoprotein in a Model of

Alzheimer's Disease.

Front. Aging Neurosci. 13:815251.

doi: 10.3389/fnagi.2021.815251
Selenomethionine Improves Mitochondrial Function by Upregulating Mitochondrial Selenoprotein in a Model of Alzheimer's Disease

by Chen, C., Chen, Y., Zhang, Z.-H., Jia, S.-Z., Chen, Y.-B., Huang, S.-L., Xu, X.-W., and Song, G.-L. (2021). Front. Aging Neurosci. 13:750921. doi: 10.3389/fnagi.2021.750921

In the published article, there was an error in affiliation 1. Instead of "College of Life Sciences and Oceanography, Shenzhen University, Shenzhen, China," it should be "Shenzhen Key Laboratory of Marine Bioresources and Ecology, College of Life Sciences and Oceanography, Shenzhen University, Shenzhen, China.”

In the published article, there was an error regarding the affiliation for Chen Chen. As well as having affiliation 1, they should also have "College of Physics and Optoelectronic Engineering, Shenzhen University, Shenzhen, China."

There was another error regarding the affiliation for Guo-Li Song. As well as having affiliation 1, they should have "Shenzhen Bay Laboratory, Shenzhen, China" and "Shenzhen-Hong Kong Institute of Brain Science-Shenzhen Fundamental Research Institutions, Shenzhen, China."

The authors apologize for this error and state that this does not change the scientific conclusions of the article in any way. The original article has been updated.

Publisher's Note: All claims expressed in this article are solely those of the authors and do not necessarily represent those of their affiliated organizations, or those of the publisher, the editors and the reviewers. Any product that may be evaluated in this article, or claim that may be made by its manufacturer, is not guaranteed or endorsed by the publisher.

Copyright $\odot 2021$ Chen, Chen, Zhang, Jia, Chen, Huang, Xu and Song. This is an open-access article distributed under the terms of the Creative Commons Attribution License (CC BY). The use, distribution or reproduction in other forums is permitted, provided the original author(s) and the copyright owner(s) are credited and that the original publication in this journal is cited, in accordance with accepted academic practice. No use, distribution or reproduction is permitted which does not comply with these terms. 